\title{
Endovascular treatment of acute ischemic stroke: the end or just the beginning?
}

\author{
Maxim Mokin, M.D., Ph.D., ${ }^{1}$ Alexander A. Khalessi, M.D., M.S., ${ }^{3}$ J Mocco, M.D., M.S., ${ }^{4}$ \\ Giuseppe Lanzino, M.D., ${ }^{5}$ Travis M. Dumont, M.D., ${ }^{1}$ Ricardo A. Hanel, M.D., Ph.D., ${ }^{6}$ \\ Demetrius K. Lopes, M.D., ${ }^{7}$ Richard D. Fessler II, M.D., ${ }^{8}$ Andrew J. Ringer, M.D., 9 \\ Bernard R. Bendok, M.D., ${ }^{10}$ Erol Veznedaroglu, M.D. ${ }^{11}$ \\ Adnan H. Siddiqui, M.D., Ph.D., ${ }^{1,2}$ L. Nelson Hopkins, M.D., ${ }^{1,2}$ \\ AND Elad I. LeVy, M.D., M.B.A.1,2
}

Departments of ${ }^{l}$ Neurosurgery and ${ }^{2}$ Radiology, School of Medicine and Biomedical Sciences, University at Buffalo, State University of New York, Buffalo, New York; ${ }^{3}$ Division of Neurosurgery, University of California, San Diego, California; ${ }^{4}$ Department of Neurosurgery, Vanderbilt University, Nashville, Tennessee; ${ }^{5}$ Departments of Neurologic Surgery and Radiology, Mayo Clinic, Rochester, Minnesota; ${ }^{6}$ Department of Neurosurgery, Mayo Clinic, Jacksonville, Florida; ${ }^{7}$ Department of Neurosurgery, Rush University Medical Center, Chicago, Illinois; ${ }^{8}$ Department of Neurosurgery, St. John Providence Health System, Detroit, Michigan; ${ }^{9}$ Department of Neurosurgery, Mayfield Clinic, University of Cincinnati, Ohio; ${ }^{10}$ Department of Neurosurgery, Northwestern University, Chicago, Illinois; and ${ }^{11}$ Department of Neurosurgery, Capital Health Institute for Neurosciences, Trenton, New Jersey

\begin{abstract}
Various endovascular intraarterial approaches are available for treating patients with acute ischemic stroke who present with severe neurological deficits. Three recent randomized trials - Interventional Management of Stroke (IMS) III, Mechanical Retrieval and Recanalization of Stroke Clots Using Embolectomy (MR RESCUE), and Synthesis Expansion: A Randomized Controlled Trial on Intra-Arterial Versus Intravenous Thrombolysis in Acute Ischemic Stroke (SYNTHESIS Expansion) - evaluated the efficacy of endovascular treatment of acute ischemic stroke and, after failing to demonstrate any significant clinical benefit of endovascular therapies, raised concerns and questions in the medical community regarding the future of endovascular treatment for acute ischemic stroke. In this paper, the authors review the evolution of endovascular treatment strategies for the treatment of acute stroke and provide their interpretation of findings and potential limitations of the three recently published randomized trials. The authors discuss the advantage of stent-retriever technology over earlier endovascular approaches and review the current status and future directions of endovascular acute stroke studies based on lessons learned from previous trials. (http://thejns.org/doi/abs/10.3171/2013.10.FOCUS13374)
\end{abstract}

KeY Words - acute ischemic stroke - endovascular procedure • large-vessel occlusion • mechanical thrombectomy • neuroimaging • stent retriever

\footnotetext{
Abbreviations used in this paper: CTA = CT angiography; ECASS = European Cooperative Acute Stroke Study; ICA = internal carotid artery; IMS = Interventional Management of Stroke; MCA = middle cerebral artery; MERCI $=$ Mechanical Embolus Removal in Cerebral Ischemia; MR RESCUE = Mechanical Retrieval and Recanalization of Stroke Clots Using Embolectomy; MRA = MR angiography; NIHSS = National Institutes of Health Stroke Scale; PROACT $=$ Prolyse in Acute Cerebral Thromboembolism; rtPA = recombinant tPA; SARIS $=$ Stent-Assisted Recanalization in acute Ischemic Stroke; SWIFT = SOLITAIRE With the Intention For Thrombectomy; tPA = tissue plasminogen activator; TREVO 2 $=$ Thrombectomy Revascularization of Large Vessel Occlusions in Acute ischemic stroke; SYNTHESIS Expansion = Synthesis Expansion: A Randomized Controlled Trial on Intra-Arterial Versus Intravenous Thrombolysis in Acute Ischemic Stroke; TICI = Thrombolysis in Cerebral Infarction; TIMI $=$ Thrombolysis in Myocardial Infarction.
}

$\mathrm{O}$ VER the last decade, various endovascular intraarterial approaches have been developed for treating patients with acute ischemic stroke presenting with severe neurological deficits. Single-arm prospective studies showed higher recanalization rates for large-vessel occlusion stroke with intraarterial approaches than with intravenous recombinant tissue plasminogen activator (rtPA).

Three long-anticipated trials (MR RESCUE ${ }^{15}$ IMS III, ${ }^{1}$ and SYNTHESIS Expansion ${ }^{3}$ ) evaluating safety and efficacy of endovascular treatment of acute ischemic stroke were published earlier this year. All three trials failed to demonstrate any significant clinical benefit of endovascular therapies. These studies immediately raised concerns and questions in the medical community regarding the fu- 
ture of endovascular treatment for acute ischemic stroke, which were reflected in electronic media and medical journals. ${ }^{2,13}$ In this paper, we review the evolution of systemic intravenous and intraarterial endovascular treatment strategies for the treatment of acute stroke, and we provide our interpretation of the findings and potential limitations of the recently published trials. . $^{1,315}$ Additionally, we discuss the current status of and future directions for endovascular acute stroke studies on the basis of lessons learned from previous trials.

\section{Intravenous Thrombolysis in Acute Ischemic Stroke}

Stroke remains the leading cause of long-term disability in the US, and approximately 795,000 people suffer from stroke each year.7 The global burden of stroke is also severe; stroke remains the second leading cause of death worldwide, showing its greatest impact in lowincome countries. ${ }^{14,17}$

In 1996, intravenous thrombolysis with rtPA received FDA approval for treatment of acute ischemic stroke within the first 3 hours of symptom onset, based on the results of the landmark National Institute of Neurological Disorders and Stroke rtPA stroke trial. ${ }^{23}$ The trial demonstrated overall greater ability of rtPA in achieving long-term favorable outcomes (assessed by functional examinations - modified Rankin scale and Barthel index-administered during a 3-month follow-up period) compared with placebo treatment, with no improvement in mortality at 3 months.

The European Cooperative Acute Stroke Study (ECASS) III was a randomized study that compared intravenous rtPA with placebo and further demonstrated the benefit of rtPA when administered within 3-4.5 hours; $52 \%$ of patients had a favorable outcome after thrombolysis compared with $45 \%$ with placebo. ${ }^{8}$ Intravenous thrombolysis performed within a 3 - to 4.5 -hour window in a carefully selected group of patients (excluding patients > 80 years, those with severity of stroke symptoms according to National Institutes of Health Stroke Scale [NIHSS] score $>25$, those with previous oral anticoagulation therapy, and those with history of stroke and diabetes, which were associated with higher risk for intracranial hemorrhage in ECASS III [although recent data suggest that intravenous thrombolysis might be beneficial even in some of these high-risk patients ${ }^{21}$ ) is currently recommended by the American Heart Association (considered Class I, Level of Evidence B).$^{10}$ However, despite the American Heart Association recommendation and positive results in ECASS III, expansion of the treatment window to 4.5 hours was not approved by the FDA. Therefore, intravenous thrombolysis within the first 3 hours of stroke symptom onset remains the only FDA-approved systemic intravenous treatment in the US.

The main advantages of intravenous thrombolysis over intraarterial endovascular treatment include low cost and simplicity and ease of administration; a stroke team can administer rtPA rapidly in the emergency department after completion of basic laboratory testing and exclusion of hemorrhage on noncontrast CT scanning of the head, without the need for an angiography suite and involvement of multiple personnel. Despite an increase in the number of acute stroke cases treated with intravenous rtPA, most patients are still excluded from receiving thrombolysis, due to delayed presentation or numerous contraindications for systemic administration of rtPA. A recent analysis of intravenous rtPA utilization in acute stroke showed a steady increase in cases from $1.2 \%$ in 2004 to $3.4 \%$ in 2009.9 Still, although that study demonstrated an almost 3 -fold increase in intravenous rtPA utilization over a 5-year period, it shows that a staggering $7 \%$ of acute stroke patients do not receive the FDA-recommended treatment despite meeting the inclusion criteria.

\section{Intravenous Thrombolysis and Large-Vessel Occlusion}

Analyses have demonstrated that the clinical efficacy of intravenous thrombolysis and its ability to achieve successful recanalization are limited in patients presenting with acute stroke due to large-vessel occlusion. This commonly includes occlusions of the following arteries: internal carotid artery (ICA), proximal middle cerebral artery (MCA) segments, vertebral artery, and basilar artery. Transcranial Doppler ultrasonography performed immediately after systemic rtPA administration showed a strong relationship between early response to thrombolysis, dramatic recovery, and occlusion site..$^{31}$ Occlusion of the ICA terminus was least likely to achieve recanalization (successful in only 6\%). Conversely, smaller, more distal occlusions had a much greater chance for successful revascularization, which increased to an impressive $44 \%$ of cases when thrombus was present within the $\mathrm{M}_{2}$ segment, whereas more proximally located MCA occlusions had successful recanalization with rtPA in only $30 \%$. A strong relationship was found between occlusion site (that is, distal MCA occlusion) and ability to achieve early dramatic neurological recovery. Computed tomography angiography (CTA) has also shown a strong impact of clot location on long-term outcomes following intravenous thrombolysis: more than $80 \%$ of treated stroke patients with a more distally located clot had good neurological outcome at 3 months. ${ }^{34}$ Proximal MCA occlusion was associated with only a $22 \%$ chance of good outcome, and none of the patients with occlusion located in the most distal segment of the ICA experienced good recovery. ${ }^{34}$

\section{Large-Vessel Occlusion as a Target for Endovascular Therapy}

Enthusiasm for endovascular catheter-assisted interventions for acute stroke was in part triggered by observations of limited recanalization rates achieved with systemic thrombolysis in patients with large-vessel occlusion. In trials of intraarterial therapies conducted in the 1990s, investigators relied on clinical evaluation alone for screening of cases in which large-vessel occlusion was suspected, because noninvasive imaging modalities (such as CTA or magnetic MR angiography [MRA]) were not widely available. Even at this early stage of emergency 


\section{Endovascular treatment of stroke}

stroke treatment it was recognized that such an approach was a poor predictor for identifying patients with largevessel occlusion.

The Prolyse in Acute Cerebral Thromboembolism (PROACT) II trial evaluated intraarterial thrombolysis with the pharmacological agent prourokinase in patients with suspected MCA occlusion. ${ }^{6}$ Clinically eligible patients $(n=474)$ were required to undergo screening catheter angiography. Of those, only a minority $(n=180)$ qualified for trial enrollment and randomization, whereas most patients $(n=294)$ were excluded simply because the suspected occlusion was not confirmed by the angiogram or was present but in a different anatomical location than originally suspected. Such a high number of patients excluded from enrollment can be explained by the relatively mild symptoms required to qualify for angiography in the trial. The minimum NIHSS score to qualify for trial enrollment in PROACT II was set at 4.

Subsequent studies have generated concerns regarding the suboptimal predictive value of the NIHSS score in identifying patients with large-vessel occlusion, especially those who present after a longer interval from symptom onset to clinical evaluation. ${ }^{25,39}$ In a prospective cohort study of 699 patients with symptoms consistent with acute ischemic stroke, CTA demonstrated significant overlap in the range of stroke severity (measured by NIHSS score) in patients with and without occlusion of a proximal vessel, confirming that NIHSS score alone is an unreliable predictor for larger vessel occlusion when selecting patients for endovascular interventions. ${ }^{18}$ The study investigators estimated that the NIHSS score threshold of 10 would result in almost equal numbers of patients with and without largevessel occlusion (meaning that almost $50 \%$ of patients with an NIHSS score of 10 would not have large-vessel occlusion but instead have stroke from other causes). Only higher NIHSS score was a reliable predictor: large-vessel occlusion was found in $90 \%$ of cases with an initial NIHSS score $\geq 16$, and an NIHSS score of 27 was $100 \%$ predictive of a proximally located occlusive lesion amenable to endovascular intervention.

\section{Evolution of Endovascular Stroke Technology}

A review of prospective trials of intraarterial stroke therapies since 1999 demonstrated a significant improvement in recanalization rates over the last 2 decades. ${ }^{4}$ The most recent trials, which included the use of stent-retriever technology, reported successful recanalization in approximately $85 \%$ of cases, compared with recanalization rates of only $50 \%$ with earlier technologies (such as pharmacological thrombolysis or mechanical thrombectomy with the Merci retriever [Stryker]). Figure 1 illustrates different endovascular approaches to stroke treatment.

Several grading systems have been developed to describe revascularization during neurointervention on the basis of cerebral angiography findings. ${ }^{38,41}$ The two most commonly used are Thrombolysis in Myocardial Infarction (TIMI) and Thrombolysis in Cerebral Infarction (TICI). The TIMI grading system was originally developed to assess the degree of cardiac reperfusion during interventions for acute myocardial infarction and subse- quently was applied to neurointerventions. ${ }^{37}$ The TICI grading system was a modification of the TIMI scale developed specifically for the intracranial circulation..$^{10}$ Grades in both systems range from 0 (no recanalization/ reperfusion) to 3 (complete recanalization/reperfusion), but TICI allows more detailed description of partial recanalization and a greater reperfusion range: none to minimal limited to the parent artery (TICI Grade 0 or 1), partial reperfusion beyond the occlusion site (TICI Grade $2 \mathrm{a}$ ), near-complete or more than $50 \%$ reperfusion beyond the occlusion site (TICI Grade 2b), or complete (TICI Grade 3). Figure 2 provides examples of cerebral angiographic runs corresponding to different degrees of the TICI scale.

Pharmacological intraarterial thrombolysis was the first endovascular strategy that was extensively studied for acute ischemic stroke treatment (Fig. 1A). In PROACT II, partial or complete recanalization occurred in $66 \%$ of cases of acute MCA occlusion treated with this approach. ${ }^{6}$ However, complete recanalization (TIMI Grade 3, which is strongly associated with the best clinical outcomes) was observed in only $19 \%$ of patients, indicating the overall poor efficacy of intraarterial pharmacological thrombolysis for these lesions. Occlusions within the ICA terminus segment (which are most resistant to treatment with intravenous thrombolysis ${ }^{34}$ ) were excluded from this study.

Mechanical thrombectomy with the Merci retriever truly revolutionized endovascular treatment of acute stroke (Fig. 1C). This device was prospectively evaluated in the Mechanical Embolus Removal in Cerebral Ischemia (MERCI) and in multi-MERCI trials in patients with intracranial occlusion of the anterior or posterior circulation, including the most challenging ICA terminus and basilar occlusions. ${ }^{35,36}$ The trials showed a strong correlation between good clinical outcome and successful recanalization. Because these studies were single arm in design, they did not directly compare outcomes of thrombectomy with the Merci retriever with other endovascular approaches.

In 2008, the FDA granted approval for a second endovascular device-the Penumbra aspiration system (Penumbra, Inc.) - which is based on the principle of clot aspiration through a reperfusion catheter coupled with mechanical breakdown of the clot with separators of various sizes (Fig. 1D). Similar to studies evaluating the Merci device, the Penumbra system was evaluated only in a single-arm prospective study, the Penumbra Pivotal Stroke trial. ${ }^{27}$ The Merci retriever and the Penumbra aspiration system were the main tools in the arsenal of stroke neurointerventionists until recently, when newer devices called stent retrievers showed superiority over earlier technology and instantly became the dominant device in the neurointerventional suite.

Stent retrievers work by temporarily deploying a stent that captures the thrombus and at the same time instantly restores blood flow to the affected brain territory by displacing the clot peripherally against the artery wall, a concept called "temporary endovascular bypass" (Fig. $1 \mathrm{E})$. The ability to obtain rapid recanalization in the setting of acute stroke was first demonstrated with permanently deployed intracranial stents in the Stent-Assisted 


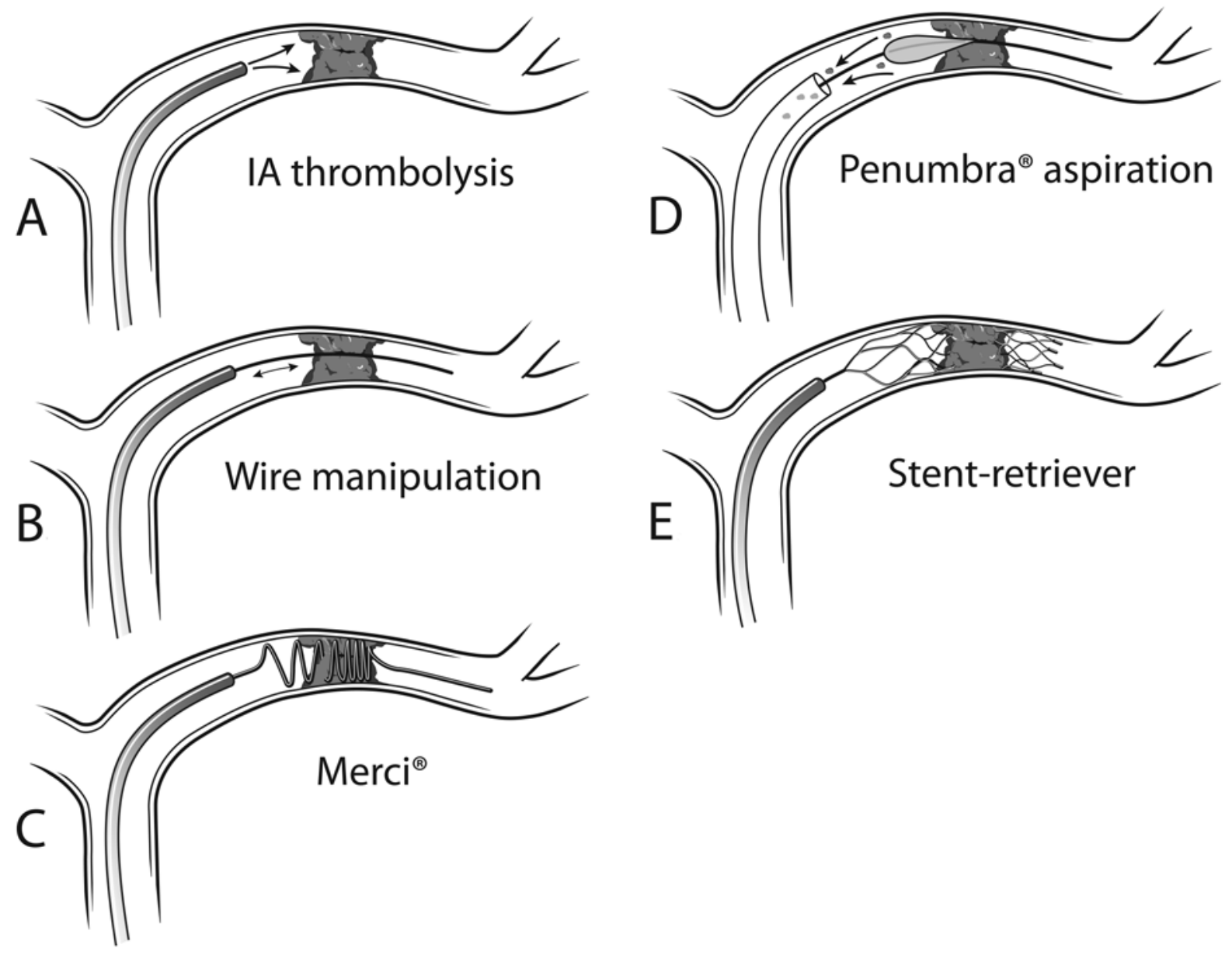

FIG. 1. Illustration of different approaches to intraarterial (IA) treatment of patients with acute stroke due to large-vessel occlusion. An intracranial vessel with occlusive thrombus is shown. A: Intraarterial pharmacological thrombolysis is done by injecting a thrombolytic agent through a microcatheter directly into the clot. B: Microwire manipulation breaks down the clot into smaller particles. C: Mechanical thrombectomy with the Merci retriever works by wrapping around and capturing the clot. D: Aspiration thrombectomy using the Penumbra aspirator and separator breaks down the clot under constant negative pressure. E: A stent retriever (Solitaire or Trevo) allows capture of the thrombus and instant restoration of blood flow. Both the stent and clot are then retrieved together. Copyright University at Buffalo Neurosurgery. Published with permission.

Recanalization in acute Ischemic Stroke (SARIS) trial, in which the median time from procedure onset to successful recanalization was only 45 minutes. ${ }^{16}$ With stent retrievers, both the thrombus and stent are retrieved after flow restoration, thus eliminating the need for the dual antiplatelet therapy that is typically required with intracranial stent implantation.

Two randomized trials comparing the Merci retriever with the Solitaire FR (Covidien) (SOLITAIRE With the Intention For Thrombectomy [SWIFT] trial) and the Trevo (Stryker) (Thrombectomy Revascularization of Large Vessel Occlusions in Acute ischemic stroke [TREVO 2] trial) stent retrievers in patients treated within 8 hours of symptom onset demonstrated higher recanalization rates and significantly improved clinical outcomes in patients treated with the stent retrievers (Table 1). ${ }^{24,33}$ In the SWIFT trial, a remarkable $58 \%$ of patients achieved good neurological outcome at 3 months, compared with only $33 \%$ of patients in the Merci group, an almost 2-fold magnitude of difference. ${ }^{33}$ At the same time, mortality rates were lower in the Solitaire group than in the Merci group
(17\% and $38 \%$, respectively). On the basis of these results, the FDA granted approval of the Solitaire FR and Trevo stent-retriever devices for treatment of stroke due to largevessel occlusion.

\section{IMS III Trial}

The IMS III trial tested the hypothesis that a combined intravenous-intraarterial approach ("bridging") to recanalization of acute stroke is superior to intravenous thrombolysis alone (Table 1). ${ }^{1}$ Intravenous rtPA had to be administered within 3 hours of stroke symptom onset. This is the largest randomized stroke trial conducted so far, with an enrollment of 900 subjects planned to demonstrate the superiority of the combined approach.

After the first 656 participants had been enrolled, the study was halted because an interim analysis demonstrated futility, that is, a low likelihood of finding a significant difference between the two treatment groups. The main finding of the trial was similar (3-month clinical outcomes in the two groups). There were no major safety 
$\mathrm{TICl} 0$

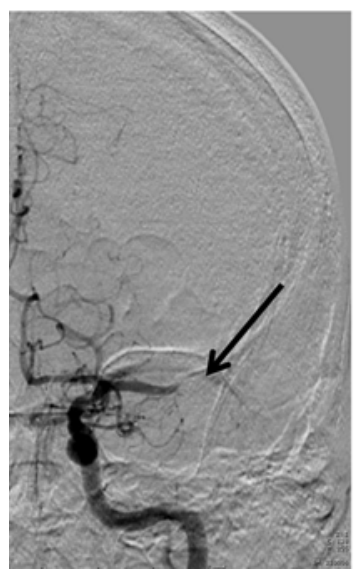

$\mathrm{TICl} 1$

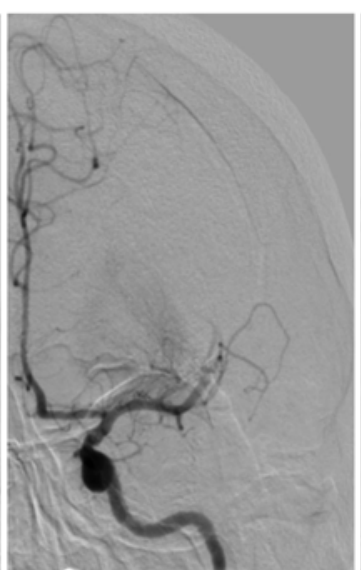

$\mathrm{TICl} 2 \mathrm{a}$

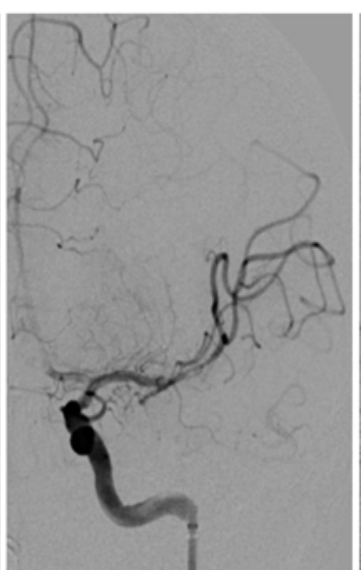

$\mathrm{TICl} 2 \mathrm{~b}$

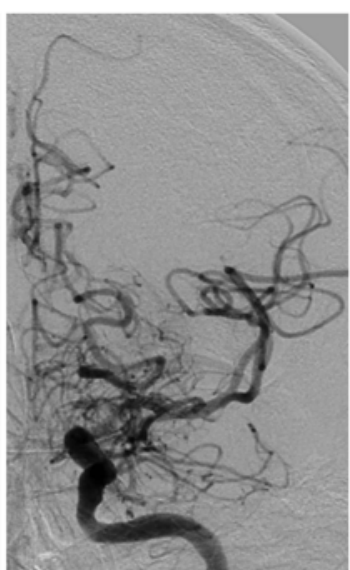

$\mathrm{TICl} 3$

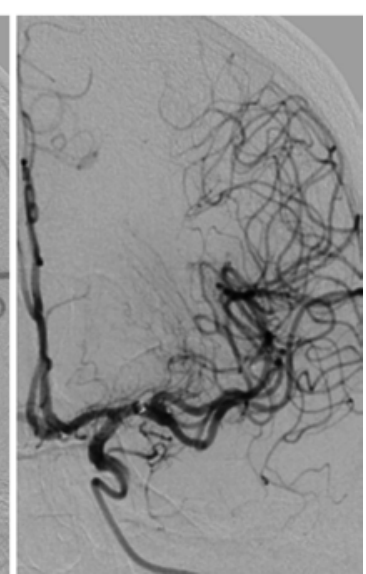

FIG. 2. Examples of the $\mathrm{TICl}$ score in a case of proximal MCA occlusion. From left to right: $\mathrm{TICI} 0$ shows no recanalization/ reperfusion of the primary occluded vessel (arrow). $\mathrm{TICl} 1$ shows partial reperfusion beyond the initial occlusion but not filling of distal MCA branches. TICl $2 \mathrm{a}$ and $\mathrm{TICl} 2 \mathrm{~b}$ correspond to partial $(<50 \%)$ and near-complete $(>50 \%$ but less than full) reperfusion beyond the occlusion site, respectively. $\mathrm{TICI} 3$ indicates complete reperfusion of the entire MCA territory.

concerns; the rates of symptomatic intracranial hemorrhage (the most feared complication of revascularization in acute ischemic stroke) and death were similar between the groups.

Documentation of large-vessel occlusion on noninvasive imaging was not incorporated in the original IMS III protocol, and an NIHSS score of 10 or higher was used for screening purposes. Only after 284 patients had been enrolled did a protocol modification allow CTA so that the investigators could exclude patients with severe neurological deficits in the absence of a target lesion amenable to endovascular intervention. As discussed earlier, the NIHSS score alone is not an accurate enough predictor of large-vessel occlusion, allowing patients without large-vessel occlusion (meaning those without a target for intraarterial therapy) to mistakenly enter the trial. This resulted in $20 \%$ of patients enrolled in the endovascular arm having no large-vessel occlusion on angiography and therefore not receiving any endovascular therapy, yet those patients were considered in the endovascular treatment arm for trial purposes. In a subgroup analysis of patients with confirmed large-vessel occlusion, intravenous rtPA plus endovascular therapy was significantly better in terms of recanalization and outcomes than intravenous rtPA alone ( $p=0.01$ ) (AM Demchuk, presented at the International Stroke Conference, 2013).

Despite the investigators' attempts to stay abreast of rapidly evolving endovascular stroke technology by incorporating new-generation devices as FDA clearance was obtained, of the 334 patients randomized to and receiving endovascular therapy, only 5 were treated with stent retrievers, that is, the technology associated with the highest reperfusion rates. Most patients in the endovascular arm received pharmacological intraarterial thrombolysis or thrombectomy with the Merci retriever, 2 approaches that are currently considered antiquated for endovascular stroke intervention.

The IMS III trial also suffered from a significant delay of more than 2 hours between initiation of intravenous
rtPA and intraarterial therapies, which was longer than in the earlier IMS I and IMS II trials and potentially diminished the clinical effect of intraarterial interventions. As expected, the shorter time from the administration of intravenous rtPA to the beginning of intervention was associated with a direction of effect toward better outcomes in IMS III. These issues raise serious concerns regarding relevance of the trial to endovascular stroke treatment as practiced today.

\section{SYNTHESIS Expansion Trial}

In the randomized SYNTHESIS Expansion trial, endovascular therapy for ischemic stroke performed within 4.5 hours of symptom onset was compared with intravenous thrombolysis alone (Table 1). ${ }^{3}$ However, unlike the IMS III trial, patients who were randomized into the endovascular arm of this trial were directly treated with intraarterial therapies (no previous "bridging" protocol). Similar to the results of IMS III, there were no significant differences in safety (rates of intracranial hemorrhage and death) or long-term outcomes between the two treatment approaches.

Again, the trial protocol did not include confirmation of large-vessel occlusion by noninvasive imaging (such as CTA or MRA) before randomization, allowing patients with strokes due to other mechanisms (such as lacunar strokes) to mistakenly enter the trial. Moreover, a minimal NIHSS threshold for study enrollment was not defined in the trial protocol.

More concerning is that intraarterial thrombolysis was allowed even in cases in which angiography showed no occlusion; rtPA was injected into the vascular territory that was presumably affected. This practice would inevitably include patients with strokes due to small-vessel disease. This population responds better to intravenous rtPA and is not considered appropriate for intraarterial therapy by most practitioners. Unfortunately, angiographic assessment of perfusion deficit pre- and postinterven- 
M. Mokin et al.

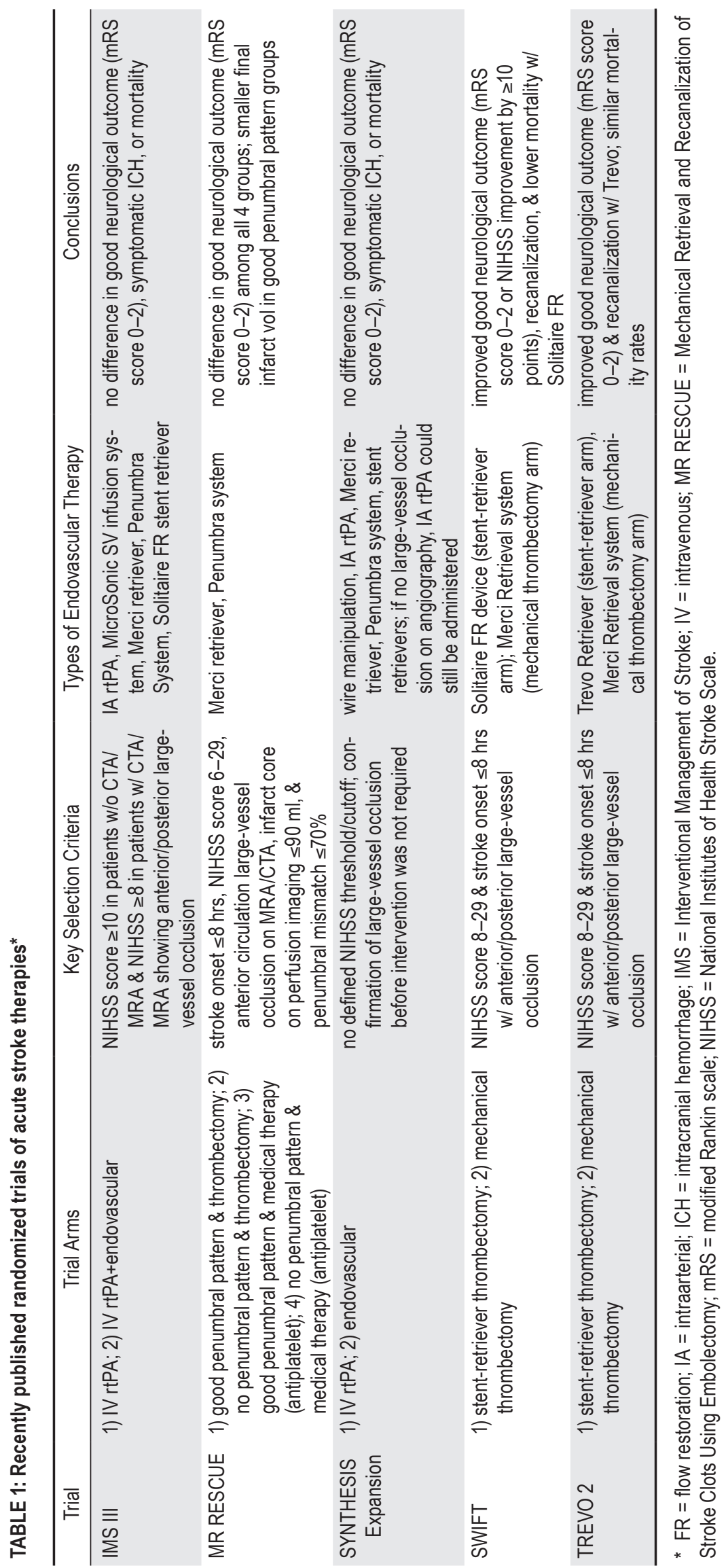




\section{Endovascular treatment of stroke}

tion was not provided in the trial. Of 181 patients in the endovascular arm, stent retrievers were used in only 23 patients (13\%); most patients in this arm were treated with wire manipulation and local thrombolysis-treatment that is no longer considered the standard for endovascular stroke interventions.

\section{MR RESCUE Trial}

The MR RESCUE trial tested the hypothesis that a favorable neuroimaging ("penumbral") pattern can identify patients likely to achieve greater benefit from endovascular treatment of acute stroke (Table 1)..$^{15}$ Patients with confirmed large-vessel occlusion were randomized to receive endovascular treatment or standard medical treatment within 8 hours of ischemic stroke symptom onset on the basis of the presence of a favorable penumbral pattern. A favorable penumbral pattern was defined as a difference between predicted brain tissue at risk (perfusion defect) and infarcted core with irreversible damage constituting up to $70 \%$ of at-risk area (diffusion defect). The final results showed similar outcomes in the two groups, arguing against the "penumbra" hypothesis in patient selection for endovascular therapies.

Randomization of patients with an infarct core volume as large as $90 \mathrm{ml}$ was permitted. Such high volumes of infarcted core are associated with poor outcomes following revascularization, which raises the argument that the design of the MR RESCUE trial allowed the inclusion of cases that potentially would not have benefited from revascularization, even if they were technically successful. ${ }^{30,40}$

The study randomized patients into 4 groups based on perfusion results (penumbral vs nonpenumbral) and type of treatment (standard medical vs endovascular), with 2034 patients in each group. Such a small number of patients in each group likely underpowered this trial, which should also be considered when interpreting the data.

It should be noted that stent retrievers were not included in the endovascular arm. An angiographically successful outcome was defined as TICI Grade 2 a or 3 revascularization. A TICI Grade 2 a corresponded to only partial reperfusion of the affected territory (Fig. 2). Only $27 \%$ of patients were able to achieve a TICI Grade $2 \mathrm{~b}$ or 3 (perfusion over two-thirds of the affected territory or full perfusion, respectively), which would indicate more robust reperfusion, arguing that effective recanalization was not achieved in most patients in the endovascular group, regardless of a penumbral imaging pattern. Distinguishing between these degrees of recanalization is clinically important. For example, partial (TICI Grade 2a) versus near-complete (TICI Grade $2 b$ or 3 ) intraarterial reperfusion has significant implications for both infarct volumes and clinical outcomes; better reperfusion rates directly correlate with smaller final infarct volume and improved clinical outcomes. ${ }^{12}$

\section{Lessons Learned}

What have we learned from these latest three trials of endovascular treatment of acute stroke? The trials confirmed a strong association between the degree of successful revascularization and improved clinical out- comes, which was demonstrated earlier by a meta-analysis of acute stroke trials that included various intravenous and intraarterial therapies. ${ }^{28}$ They confirmed the importance of achieving rapid reperfusion and that minimizing delays from symptom onset to reperfusion time can lead to better patient outcomes. Finally, the trials demonstrated similar safety profiles of intravenous and intraarterial reperfusion therapies, including intracranial hemorrhage rates, which were thought to be much higher with endovascular approaches in the past.

However, these three trials do not demonstrate failure of endovascular therapy for acute stroke. They do not reflect modern standards of intraarterial revascularization in patients with acute stroke. Evaluation for large-vessel occlusion with noninvasive imaging is currently recommended by many practitioners for all patients with acute stroke considered for emergency endovascular interventions, ${ }^{11}$ yet the IMS III ${ }^{1}$ and SYNTHESIS Expansion ${ }^{3}$ trials did not require such screening prior to enrollment and randomization.

A subgroup analysis of the IMS III trial that included only patients with confirmed large-vessel occlusion showed a benefit of endovascular therapy over intravenous rtPA alone. ${ }^{3}$ Although consideration of acute stroke patients for intraarterial therapies based on NIHSS score and noncontrast brain $\mathrm{CT}$ results alone might have a benefit of minimizing timing from symptom onset to reperfusion, it cannot be an acceptable standard in clinical trials. Randomized controlled trials can be considered the benchmark when comparing safety and efficacy of therapeutic interventions only when the treatment groups are appropriately selected to ensure the trial's validity and accuracy. Moreover, with modern imaging technology, CTA can be rapidly performed at the time of the first noncontrast CT scan without further delays and with a relatively low radiation and contrast dose. Current acute stroke management guidelines support the use of CT or perfusion MRI along with diffusion-weighted MRI for estimation of the size of irreversibly infarcted area and ischemic penumbra, especially when considering patients for endovascular therapies beyond the standard time window for intravenous thrombolysis. ${ }^{11}$

The three trials in question also underscore practical issues encountered when evaluating rapidly evolving technology. Stent retrievers have been shown to be more effective in achieving revascularization than previous mechanical and pharmacological devices. Nevertheless, the proportion of patients treated with stent retrievers in the recent three trials was so small that it would be deceptive to interpret the results of those trials as a reflection of modern practice. In combining these three trials together, only $28(5 \%)$ of 579 patients treated with various endovascular approaches were treated with stent retrievers. At the most recent American Academy of Neurology annual meeting, results from the North American Solitaire acute stroke registry were presented, specifically focusing on cases of acute stroke treated with intravenous thrombolysis followed by Solitaire FR stent retrieval (OO Zaidat, presented at the American Academy of Neurology Annual Meeting, 2013). Outcomes from this IMS III-like cohort were compared with results from the intravenous 
thrombolysis-only group in the IMS III trial, and better clinical outcomes were found in patients treated with a combination of intravenous rtPA and stent retrievers. Although comparison of these two studies has limitations and should be interpreted with caution, it suggests that recent technology-which was not adequately studied in the trials in question-may play a critical role in acute stroke endovascular therapy.

The current uncertainty regarding the role of endovascular therapy in acute stroke is reminiscent of the evolution of percutaneous cardiac interventional procedures for myocardial infarction; after numerous trials and tribulations, it was not until newer-generation devices became available that the interventional approach became the accepted treatment of acute myocardial infarction. Although coronary and cerebral reperfusion therapies share common patterns, cerebral reperfusion therapies are advancing at a slower pace. ${ }^{26}$ The use of stent-retriever technology is the first treatment capable of showing higher and more complete reperfusion rates, comparable to coronary interventions for myocardial infarction. ${ }^{32}$

What lessons have we learned to ensure that future stroke trials will definitively answer the questions regarding the value of intraarterial stroke therapies? To ensure appropriate selection of patients, all potential candidates should undergo a baseline CTA or MRA evaluation to document the presence and extent of large-vessel occlusion. Clot characteristics, including length and composition, are important indicators of recanalization after intravenous thrombolysis and can be used as biomarkers in selecting patients with a higher likelihood of benefit from the intraarterial approach. ${ }^{22,29}$ All attempts should be made to minimize delays from stroke symptom onset to achievement of successful reperfusion. Stent retrievers should be the first-line treatment device used in clinical trials, given their clear benefit in achieving faster, more robust reperfusion and improved clinical outcomes. Newer-generation devices such as large-bore catheters delivered intracranially with direct clot aspiration through direct carotid artery puncture techniques may further enhance clot retrieval speed and efficacy. Finally, advances in imaging technology involving perfusion analyses using CT or MR methodologies with more reasonable "favorable penumbral patterns" are constantly improving our ability to identify those patients who are more likely to benefit from acute stroke interventions. ${ }^{5}$

Several ongoing clinical trials have incorporated such key selection points in their design. For example, The Randomized, Concurrent Controlled Trial to Assess the Penumbra System's Safety and Effectiveness in the Treatment of Acute Stroke (THERAPY) is evaluating the efficacy of the Penumbra System as adjunctive therapy to intravenous thrombolysis in the setting of arterial occlusion with a large clot burden (clot length $>8 \mathrm{~mm}$ ) to identify patients who are unlikely to respond to intravenous thrombolysis alone (ClinicalTrials.gov identifier NCT01429350). The Solitaire FR With the Intention For Thrombectomy as Primary Endovascular Treatment for Acute Ischemic Stroke (SWIFT-PRIME) trial is examining whether combining Solitaire FR stent-retriever thrombectomy with intravenous thrombolysis improves clinical outcomes (ClinicalTrials.gov identifier NCT01657461). Patients with a favorable imaging pattern based on an MR or a CT perfusion study are selected for this trial, and the protocol is designed to minimize the time interval between imaging and angiography.

The PerfusiOn Imaging Selection of Ischemic STroke PatIents for EndoVascular ThErapy (POSITIVE) trial will be launched soon (ClinicalTrials.gov identifier NCT01852201). Instead of testing a specific endovascular device, it will allow utilization of multiple endovascular approaches, including stent retrievers. Device use alone or in combination with other devices will be determined by the treating interventionist on a case-by-case basis and will be compared with standard medical therapy with antithrombotic agents in patients with acute stroke due to large-vessel occlusion. The trial will include patients with a favorable perfusion pattern within 12 hours of stroke onset, significantly extending the time window for patient enrollment in comparison with previous trials. Treatment of strokes with unknown time of onset or socalled "wake-up" stroke is another promising direction of endovascular stroke therapy. Emerging data suggest that intravenous thrombolysis in carefully selected wakeup stroke patients might be safe and effective. ${ }^{19,20}$ Such patients, most of whom are excluded from intravenous thrombolysis in daily clinical practice, may prove to be excellent candidates for future endovascular stroke trials.

We encourage investigators from all involved fields to join efforts in developing more effective strategies for the treatment of acute stroke. Endovascular approaches to stroke treatment will continue to evolve and so will continue to improve our capabilities to help patients suffering from this devastating condition.

\section{Acknowledgments}

We thank Paul H. Dressel, B.F.A., for preparation of the illustrations and Debra J. Zimmer for editorial assistance.

\section{Disclosure}

Dr. Dumont, Dr. Fessler, Dr. Khalessi, and Dr. Veznedaroglu report no disclosures or potential conflicts of interest. The remaining authors report the following relationships. Dr. Bendok: research grants from MicroVention, the Erika Keeney foundation, and the NIH (not related to this submission). Dr. Hanel: consultant for Codman, Covidien, and Stryker; and investor in Blockade Medical. Dr. Hopkins: grant/research support from Toshiba; consultant to Abbott, Boston Scientific, Cordis, Micrus, and Silk Road; financial interests in AccessClosure, Augmenix, Boston Scientific, Claret Medical, Endomation, Micrus, and Valor Medical; board/trustee/officer position with Access Closure and Claret Medical; Abbott Vascular's speakers' bureau; honoraria from Bard, Boston Scientific, Cleveland Clinic, Complete Conference Management, Cordis, Memorial Health Care System, and the SCAI. Dr. Lanzino: consultant to ev3/ Covidien. Dr. Levy: research grant support, other research support (devices), and honoraria from Boston Scientific and research support from Codman \& Shurtleff, Inc. and ev3/Covidien Vascular Therapies; ownership interests in Intratech Medical Ltd. and Mynx/Access Closure; consultant on the board of Scientific Advisors to Codman \& Shurtleff, Inc.; consultant per project and/or per hour for Codman \& Shurtleff, Inc., ev3/Covidien Vascular Therapies, and TheraSyn Sensors, Inc.; and fees for carotid stent training from Abbott Vascular and ev3/Covidien Vascular Therapies. Dr. Lopes: stock options 


\section{Endovascular treatment of stroke}

in Penumbra; advisory board for Covidien, Siemens, and Stryker; and proctor for Covidien. Dr. Mocco: consultant to Lazarus Effect, Reverse Medical, and Pulsar; investor in Blockade Medical. Dr. Mokin: Toshiba research fellowship. Dr. Ringer: consulting fees from Stryker, Microvention, and Covidien/ev3 but has not received any fees or honoraria for any product discussed in this manuscript. Dr. Siddiqui: research grants from NIH (co-investigator: NINDS Grant No. 1R01NS064592-01A1), University at Buffalo (neither grant applies to the present submission); financial interests in Hotspur, Intratech Medical, StimSox, Valor Medical, and Blockade Medical; consultant for Codman \& Shurtleff, Inc., Concentric Medical, Covidien Vascular Therapies, GuidePoint Global Consulting, Penumbra, Stryker Neurovascular, and Pulsar Vascular; speakers' bureaus for Codman \& Shurtleff and Genentech; serves on National Steering Committees for Penumbra 3D Separator Trial and Covidien SWIFT PRIME Trial; advisory board for Codman \& Shurtleff and Covidien Vascular Therapies; honoraria from American Association of Neurological Surgeons' courses, Annual Peripheral Angioplasty and All That Jazz Course, Penumbra, and from Abbott Vascular and Codman \& Shurtleff, Inc. for training other neurointerventionists in carotid stenting and for training physicians in endovascular stenting for aneurysms.

Author contributions to the study and manuscript preparation include the following. Conception and design: Levy, Mokin, Khalessi, Mocco, Hopkins. Acquisition of data: all authors. Analysis and interpretation of data: Levy, Mokin, Khalessi, Mocco, Lanzino, Dumont, Hanel, Fessler, Ringer, Bendok, Veznedaroglu, Siddiqui, Hopkins. Drafting the article: Mokin. Critically revising the article: all authors. Reviewed submitted version of manuscript: all authors.

\section{References}

1. Broderick JP, Palesch YY, Demchuk AM, Yeatts SD, Khatri P, Hill MD, et al: Endovascular therapy after intravenous t-PA versus t-PA alone for stroke. N Engl J Med 368:893-903, 2013

2. Chimowitz MI: Endovascular treatment for acute ischemic stroke-still unproven. N Engl J Med 368:952-955, 2013

3. Ciccone A, Valvassori L, Nichelatti M, Sgoifo A, Ponzio M, Sterzi R, et al: Endovascular treatment for acute ischemic stroke. N Engl J Med 368:904-913, 2013

4. Fargen KM, Meyers PM, Khatri P, Mocco J: Improvements in recanalization with modern stroke therapy: a review of prospective ischemic stroke trials during the last two decades. J Neurointerv Surg 5:506-511, 2013

5. Fisher M, Albers GW: Advanced imaging to extend the therapeutic time window of acute ischemic stroke. Ann Neurol 73: 4-9, 2013

6. Furlan A, Higashida R, Wechsler L, Gent M, Rowley H, Kase $\mathrm{C}$, et al: Intra-arterial prourokinase for acute ischemic stroke. The PROACT II study: a randomized controlled trial. JAMA 282:2003-2011, 1999

7. Go AS, Mozaffarian D, Roger VL, Benjamin EJ, Berry JD, Borden WB, et al: Executive summary: heart disease and stroke statistics-2013 update: a report from the American Heart Association. Circulation 127:143-152, 2013

8. Hacke W, Kaste M, Bluhmki E, Brozman M, Dávalos A, Guidetti D, et al: Thrombolysis with alteplase 3 to 4.5 hours after acute ischemic stroke. N Engl J Med 359:1317-1329, 2008

9. Hassan AE, Chaudhry SA, Grigoryan M, Tekle WG, Qureshi AI: National trends in utilization and outcomes of endovascular treatment of acute ischemic stroke patients in the mechanical thrombectomy era. Stroke 43:3012-3017, 2012

10. Higashida RT, Furlan AJ, Roberts H, Tomsick T, Connors B, Barr J, et al: Trial design and reporting standards for intra-arterial cerebral thrombolysis for acute ischemic stroke. Stroke 34:e109-e137, 2003

11. Jauch EC, Saver JL, Adams HP Jr, Bruno A, Connors JJ, De- maerschalk BM, et al: Guidelines for the early management of patients with acute ischemic stroke: a guideline for healthcare professionals from the American Heart Association/American Stroke Association. Stroke 44:870-947, 2013

12. Jayaraman MV, Grossberg JA, Meisel KM, Shaikhouni A, Silver B: The clinical and radiographic importance of distinguishing partial from near-complete reperfusion following intra-arterial stroke therapy. AJNR Am J Neuroradiol 34: 135-139, 2013

13. Jeffrey S: No advantage for endovascular stroke treatment over IV tPA. Medscape. (http://www.medscape.com/viewarticle/778919) [Accessed November 14, 2013]

14. Johnston SC, Mendis S, Mathers CD: Global variation in stroke burden and mortality: estimates from monitoring, surveillance, and modelling. Lancet Neurol 8:345-354, 2009

15. Kidwell CS, Jahan R, Gornbein J, Alger JR, Nenov V, Ajani Z, et al: A trial of imaging selection and endovascular treatment for ischemic stroke. N Engl J Med 368:914-923, 2013

16. Levy EI, Siddiqui AH, Crumlish A, Snyder KV, Hauck EF, Fiorella DJ, et al: First Food and Drug Administration-approved prospective trial of primary intracranial stenting for acute stroke: SARIS (stent-assisted recanalization in acute ischemic stroke). Stroke 40:3552-3556, 2009

17. Lozano R, Naghavi M, Foreman K, Lim S, Shibuya K, Aboyans V, et al: Global and regional mortality from 235 causes of death for 20 age groups in 1990 and 2010: a systematic analysis for the Global Burden of Disease Study 2010. Lancet 380:2095-2128, 2012 (Erratum in Lancet 381:628, 2013)

18. Maas MB, Furie KL, Lev MH, Ay H, Singhal AB, Greer DM, et al: National Institutes of Health Stroke Scale score is poorly predictive of proximal occlusion in acute cerebral ischemia. Stroke 40:2988-2993, 2009

19. Manawadu D, Bodla S, Jarosz J, Keep J, Kalra L: A case-controlled comparison of thrombolysis outcomes between wakeup and known time of onset ischemic stroke patients. Stroke 44:2226-2231, 2013

20. Manawadu D, Bodla S, Keep J, Jarosz J, Kalra L: An observational study of thrombolysis outcomes in wake-up ischemic stroke patients. Stroke 44:427-431, 2013

21. Mazya MV, Lees KR, Markus R, Roine RO, Seet RC, Wahlgren N, et al: Safety of intravenous thrombolysis for ischemic stroke in patients treated with warfarin. Ann Neurol [epub ahead of print], 2013

22. Miller TS, Brook AL, Riedel CH, Hirsch JA, Yoo AJ: Expanding the role of NCCT in acute stroke imaging: thrombus length measurement and its potential impact on current practice. J Neurointerv Surg [epub ahead of print], 2013

23. National Institute of Neurological Disorders and Stroke rt-PA Stroke Study Group: Tissue plasminogen activator for acute ischemic stroke. N Engl J Med 333:1581-1587, 1995

24. Nogueira RG, Lutsep HL, Gupta R, Jovin TG, Albers GW, Walker GA, et al: Trevo versus Merci retrievers for thrombectomy revascularisation of large vessel occlusions in acute ischaemic stroke (TREVO 2): a randomised trial. Lancet 380: 1231-1240, 2012

25. Olavarría VV, Delgado I, Hoppe A, Brunser A, Cárcamo D, Díaz-Tapia V, et al: Validity of the NIHSS in predicting arterial occlusion in cerebral infarction is time-dependent. Neurology 76:62-68, 2011

26. Patel RD, Saver JL: Evolution of reperfusion therapies for acute brain and acute myocardial ischemia: a systematic, comparative analysis. Stroke 44:94-98, 2013

27. Penumbra Pivotal Stroke Trial Investigators: The Penumbra Pivotal Stroke Trial: safety and effectiveness of a new generation of mechanical devices for clot removal in intracranial large vessel occlusive disease. Stroke 40:2761-2768, 2009

28. Rha JH, Saver JL: The impact of recanalization on ischemic stroke outcome: a meta-analysis. Stroke 38:967-973, 2007

29. Riedel CH, Zimmermann P, Jensen-Kondering U, Stingele R, 
Deuschl G, Jansen O: The importance of size: successful recanalization by intravenous thrombolysis in acute anterior stroke depends on thrombus length. Stroke 42:1775-1777, 2011

30. Sanák D, Nosál' V, Horák D, Bártková A, Zelenák K, Herzig $\mathrm{R}$, et al: Impact of diffusion-weighted MRI-measured initial cerebral infarction volume on clinical outcome in acute stroke patients with middle cerebral artery occlusion treated by thrombolysis. Neuroradiology 48:632-639, 2006

31. Saqqur M, Uchino K, Demchuk AM, Molina CA, Garami Z, Calleja S, et al: Site of arterial occlusion identified by transcranial Doppler predicts the response to intravenous thrombolysis for stroke. Stroke 38:948-954, 2007

32. Saver JL: The 2012 Feinberg Lecture: treatment swift and treatment sure. Stroke 44:270-277, 2013

33. Saver JL, Jahan R, Levy EI, Jovin TG, Baxter B, Nogueira $\mathrm{RG}$, et al: Solitaire flow restoration device versus the Merci Retriever in patients with acute ischaemic stroke (SWIFT): a randomised, parallel-group, non-inferiority trial. Lancet 380: $1241-1249,2012$

34. Sillanpää N, Saarinen JT, Rusanen H, Elovaara I, Dastidar P, Soimakallio S: Location of the clot and outcome of perfusion defects in acute anterior circulation stroke treated with intravenous thrombolysis. AJNR Am J Neuroradiol 34:100-106, 2013

35. Smith WS, Sung G, Saver J, Budzik R, Duckwiler G, Liebeskind DS, et al: Mechanical thrombectomy for acute ischemic stroke: final results of the Multi MERCI trial. Stroke 39: 1205-1212, 2008

36. Smith WS, Sung G, Starkman S, Saver JL, Kidwell CS, Gobin
YP, et al: Safety and efficacy of mechanical embolectomy in acute ischemic stroke: results of the MERCI trial. Stroke 36:1432-1438, 2005

37. TIMI Study Group: The Thrombolysis in Myocardial Infarction (TIMI) trial-Phase I findings. N Engl J Med 312:932936, 1985

38. Tomsick T: TIMI, TIBI, TICI: I came, I saw, I got confused. AJNR Am J Neuroradiol 28:382-384, 2007

39. Uchino K: Does stroke severity predict presence of arterial occlusion? Time will tell. Neurology 76:14-15, 2011

40. Yoo AJ, Verduzco LA, Schaefer PW, Hirsch JA, Rabinov JD, González RG: MRI-based selection for intra-arterial stroke therapy: value of pretreatment diffusion-weighted imaging lesion volume in selecting patients with acute stroke who will benefit from early recanalization. Stroke 40:2046-2054, 2009

41. Zaidat OO, Lazzaro MA, Liebeskind DS, Janjua N, Wechsler L, Nogueira RG, et al: Revascularization grading in endovascular acute ischemic stroke therapy. Neurology 79 (13 Suppl 1):S110-S116, 2012

Manuscript submitted August 22, 2013.

Accepted October 15, 2013.

Please include this information when citing this paper: DOI: 10.3171/2013.10.FOCUS13374.

Address correspondence to: Elad I. Levy, M.D., M.B.A., University at Buffalo Neurosurgery, 100 High St., Ste. B4, Buffalo, NY 14203.email: elevy@ubns.com. 